\title{
Acesso aberto às publicações científicas: vantagens, políticas e advocacy ${ }^{1}$
}

\author{
Open Access to scientific publications: benefits, policy and advocacy
}

\author{
Ariadne Chloe Furnival \\ Doutor em Política Científica e Tecnológica, UNICAMP \\ Professora do Programa de Pós-graduação em Ciência, Tecnologia e Sociedade UFSCar \\ E-mail: chloeufscar@gmail.com \\ Bill Hubbard \\ Mestre em Inteligência Artificial, Kingston Polytechnic, Inglaterra \\ Diretor do Centre for Research Communications, Universidade de Nottingham, Inglaterra \\ E-mail: bill.hubbard@ nottingham.ac.uk
}

\begin{abstract}
Resumo
O movimento de Acesso Aberto (AA) entende os modos de AA de disseminar a pesquisa científica como o futuro da comunicação científica. Os proponentes deste movimento têm, nos últimos 10 anos, realizado pesquisa sistemática para demonstrar como o AA pode sensivelmente beneficiar pesquisadores, instituições e, também, a sociedade. Ainda assim, o número de artigos de pesquisa depositados nos repositórios institucionais (RIs) se mantém relativamente baixo (não mais que $15 \%$ do total de publicações), possível reflexo de preocupações e mitos em torno do AA que contradiz sua realidade. As políticas de AA têm sido introduzidas em vários níveis para encorajar a adesão de pesquisadores às práticas nesse âmbito, mas pode haver outras dimensões culturais e institucionais que interfiram na efetividade destas práticas. Ao abordar aspectos destes fenômenos, este trabalho finaliza com uma discussão em torno da promoção - advocacy, no inglês - de AA nas organizações, e se esta deveria ser a jusante, na forma de campanhas informacionais, ou a montante, na forma de ações dos órgãos de gestão. Procuramos, assim, contribuir com a discussão destas questões na esfera de AA, trazendo dentro do debate elementos da literatura da sociologia da ciência e da teoria de comportamento nas organizações que possibilitem elucidar alguns aspectos da postura de pesquisadores diante o AA.
\end{abstract}

Palavras-chave: Acesso aberto; Publicação científica; Repositório institucional; Advocacy; . Normas científicas.

\begin{abstract}
The Open Access (OA) movement regards OA modes of disseminating research as the unequivocal future of scholarly communication. Proponents of the movement itself have, over the last ten years, carried out systematic research to show how OA can tangibly benefit researchers, institutions and society at large. Even so, the number of research papers being uploaded to OA institutional repositories remains relatively low, a possible reflection of concerns about OA which often contradict the facts. Policies for OA have been introduced to encourage author uptake, and these are also discussed here. Briefly delineating aspects of these phenomena, this paper will then move on to outline and discuss advocacy for OA in organisations, and whether this should be "downstream", in the form of informational campaigns, or "upstream", in the form of top-down change management. This paper seeks to make a contribution to these issues in the OA sphere, by brining into the debate strands from the literature of the sociology of science and management science that will hopefully elucidate aspects of author reactions to $\mathrm{OA}$, and the perceived changes that its adoption gives rise to.
\end{abstract}

Keywords: Open access; Scientific publication; Institutional repository; Advocacy; Scientific norms.

\footnotetext{
${ }^{1}$ Partes deste artigo foram desenvolvidas pela primeira autora na elaboração do relatório Deliverable D 3.3 do Projeto NECOBELAC (2010), enquanto ela exercia o cargo de "Open Access Adviser - Europe", no Centre for Research Communications, University of Nottingham, Inglaterra, em 2010.
}

InCID: R. Ci. Inf. e Doc., Ribeirão Preto, v. 2, n. 2, p. 160-177, jul./dez. 2011. 


\section{Introdução}

Desde que o conceito de Acesso Aberto ou Acesso Livre (o qual designaremos simplesmente com a sigla AA) foi consagrado no Budapest OA Initiative (2002), na Bethesda Statement on Open Access Publishing (2003) e na Declaração de Berlin sobre Acesso Aberto ao Conhecimento nas Ciências e Humanidades (2003) - as três declarações juntas denominados como os “3 B's” por Peter Suber, um dos proponentes mais ativos e importantes do AA no mundo -, tem havido uma verdadeira proliferação de projetos promovendo o AA como $o$ modo mais eficaz para a comunicação científica. Recentemente, um número substancial de projetos de AA tem proporcionado a criação e o apoio de repositórios institucionais (RIs) ou disciplinares nos quais os pesquisadores são encorajados a depositarem seus preprints e postprints (versões de artigos pré- e pós-revisão por pares e subsequente publicação). Este modo de publicação AA, centrado em repositórios, é conhecido como a "via verde" ("green OA"). Outros projetos concentraram na promoção de modelos de negócios alternativos de publicação, que incluem periódicos totalmente de acesso aberto ("AA dourado") ${ }^{2}$ ou modelos "híbridos", com o autor (ou, mais especificamente, sua instituição ou órgão de fomento da pesquisa) pagando pela publicação do artigo.

Os proponentes deste modelo reconhecem cada vez mais a necessidade de atacar o trabalho menos técnico, mas igualmente formidável da promoção e incentivo do AA. Apreciase hoje que a aceitação das opções de disseminação em AA para os resultados da pesquisa e o uso dos repositórios requerem, sobretudo, uma mudança no comportamento de pesquisadores da comunidade científica junto com a existência de procedimentos institucionais normativos, como mandatos para o autoarquivamento em RIs, além de outros que favoreçam condições para uma aceitação de AA de modo mais "voluntário". O conjunto de atividades que tem como objetivo a promoção de modos de disseminação em AA e o encorajamento de pesquisadores e outros stakeholders (protagonistas) relevantes a incorporarem tais modos nos seus fluxos de trabalho existentes é usualmente denominado "advocacy" - termo que se assemelha a "promoção" no português, mas que abrange atividades que podem, inclusive, parecer um tipo de lobbying "pela causa". O trabalho de advocacy visa a eventual incorporação não problemática das práticas de AA de disseminação nos fluxos de trabalho acadêmico já existentes e, por isso, os esforços de advocacy também englobam a necessidade

\footnotetext{
${ }^{2}$ Ver Harnard et al. (2004) para saber mais sobre as vias "verde" e "dourada" de AA. Para uma lista de revistas em AA, ver o Directory of Open Access Journals (DOAJ) em: <http://www.doaj.org/>.

InCID: R. Ci. Inf. e Doc., Ribeirão Preto, v. 2, n. 2, p. 160-177, jul./dez. 2011.
} 
de recrutar o apoio de administradores acadêmicos (como, por exemplo, pró-reitores de pesquisa e de bibliotecários em cargos de direção ${ }^{3}$ ).

Reconhecendo que tais dimensões do movimento AA e sua evolução representam a próxima "frente" para ganhar aprovação e adesão, divulgaram-se, nos últimos anos, os resultados de projetos de pesquisa sobre AA que têm como enfoque os aspectos relativos à economia da publicação nesse âmbito e mudanças dos modelos de negócios (business models) na publicação científica, as políticas (policies) de AA, os mandatos a favor do AA pelos órgãos de fomento à pesquisa e as atitudes dos autores em relação ao AA (ANTELMAN, 2004; HOUGHTON et al., 2009; NICHOLAS; HUNTINGTON; ROWLANDS, 2005; SWAN; BROWN, 2005; SWAN, 2006).

Apesar desta verdadeira proliferação de pesquisa em, e promoção do, AA, é inegável que muitos RIs e disciplinares são escassamente povoados com publicações em texto completo, seja em forma de preprint, seja em forma de postprint: Stevan Harnard, outro importante ativista no movimento a favor do AA, cita, em vários momentos, a cifra de que apenas $15 \%$ do total dos 2,5 milhões de artigos publicados anualmente são depositados em RIs (HARNARD, 2006). Resta a questão do por quê isto ocorre. Assim, com base no corpo de literatura hoje substancial sobre o AA, junto com outros fios relevantes da literatura dos estudos sociais da ciência e, ainda, das teorias de comportamento de atores institucionais, este artigo pretende tecer algumas considerações na direção de responder a esta questão.

\section{As Vantagens do AA}

Os pesquisadores científicos na academia trabalham de acordo com o ditado "publique ou pereça" (publish or perish) e, muitas vezes, querem saber se sua pesquisa publicada tem surtido algum efeito positivo na sua comunidade de pares para contribuir com o avanço da pesquisa no seu campo. Tal repercussão, ou seja, o número de vezes que o artigo relatando a pesquisa é citado é usualmente referido como o fator de impacto ("Impact Factor" - IF). O termo foi cunhado por Eugene Garfield do Institute of Scientific Information (ISI) em 1955 para se referir à formulação de um índice de citações que "avaliaria a importância de um trabalho particular e seu impacto na literatura e pensamento do período" (GARFIELD, 1955,

\footnotetext{
${ }^{3}$ Hélio Kuramoto, por exemplo, em seu blog sobre o AA, elogia a bibliotecária Dra. Angélica C.D. Miranda, da Universidade Federal do Rio Grande, relatando que ela batalhou para a implementação de uma política de acesso livre para aquela universidade, o primeiro mandato de AA no Brasil (KURAMOTO, 1 de junho, 2011). InCID: R. Ci. Inf. e Doc., Ribeirão Preto, v. 2, n. 2, p. 160-177, jul./dez. 2011.
} 
p. 469). Hoje o IF é utilizado para ranquear os periódicos, além de avaliar e ranquear as instituições e seus pesquisadores, apesar dos alertas do próprio autor contra o uso do fator de impacto como um substituto da medida da "qualidade" de pesquisa.

Os proponentes do AA têm pesquisado seu efeito positivo no número de citações de artigos, o que levou ao surgimento do conceito da "Vantagem de citação de AA" ("Open Access Citation Advantage" - a OACA). Uma vez que o fator de impacto é hoje amplamente respeitado no sistema de comunicação científica, a realidade do OACA pode ser usada como um argumento convincente para promover o AA entre pesquisadores (SWAN, 2010). Esse efeito positivo do AA nas citações de artigos tem sido comprovado, como a quantidade substancial da literatura sobre o assunto atesta. Hitchcock, por exemplo, mantem uma bibliografia que atualiza de modo contínuo, sobre o efeito de AA no número de downloads de artigos. Já em 2001, Lawrence publicou, na revista Nature, o primeiro estudo sobre o OACA usando dados originais e comparando artigos, publicados entre 1989 e 2000 em ciência da computação e disciplinas relacionadas, publicamente disponíveis online (entendido por ele como sinônimos de artigos em AA) com artigos não online. Os resultados demonstraram que "a média de citações para os artigos não online é 2,74 e para artigos online, de 7,03, um aumento de 157\%" (LAWRENCE, 2001, tradução nossa) - em termos globais, a OACA seria o dobro. O estudo de Antelman (2004) revelou que o aumento relativo em citações para os artigos em AA foi de 45\% para a Filosofia, 51\% na Engenharia elétrica e eletrônica, $86 \%$ na Ciência Política e 91\% em Matemática. Ademais, os resultados de Hajjem et al. (2005) revelam que para 10 disciplinas em 10 anos, a vantagem de citação em AA - a OACA - pode ser verificada: para uma citação havia uma OACA de 16\%, para um número entre 4 e 7 citações uma OACA de $22 \%$, e para mais de 16 citações, uma OACA de $10 \%$.

Em suma, existe evidência substancial para comprovar que há, sim, uma vantagem de citação dos artigos disponíveis em AA. Na medida em que o fator de impacto e as citações ainda têm prestígio entre a comunidade científica, a OACA constitui um argumento forte a favor de AA, seja via publicação em revistas AA, seja via submissão de artigos num repositório institucional. Ainda assim, deveríamos ser cautelosos para não cair no argumento de que, se não houver uma perceptível "vantagem de citação de AA" (OACA), então o AA em si não tem valor. O AA é uma tendência crescente porque é visto como uma forma justa de fazer os resultados de pesquisa - em grande parte financiada por verba pública abertamente disponíveis na sociedade: a OACA constitui apenas uma entre outras vantagens de AA.

InCID: R. Ci. Inf. e Doc., Ribeirão Preto, v. 2, n. 2, p. 160-177, jul./dez. 2011. 
Entre estas outras vantagens de AA está o aumento na visibilidade das pesquisas de uma dada instituição. Este é o caso para o "AA verde", ou seja, o autoarquivamento de versões de artigos em RIs. Como Swann e Carr (2008, p.33) observam:

\footnotetext{
Quase toda instituição com um repositório cita isto como o motivo por tê-lo criado, de acordo com nossa pequena enquete de repositórios europeus (no prelo). Certamente, o repositório é o veículo ideal para fazer os trabalhos e resultados de uma instituição visível. Dependendo das páginas web de uma instituição, não é satisfatório (tradução nossa).
}

Com o ranking web das universidades se tornando, mundialmente, cada vez mais uma medida aceitável da visibilidade e do impacto potencial de uma universidade (ver, por exemplo, o projeto Webometrics ${ }^{4}$ ), o trafego ao RI da universidade para baixar artigos constituirá um papel de crescente importância na produção de tais medidas. Ouvimos em uma apresentação, um Chief Information Officer de uma das mais importantes universidades do Reino Unido, alegar que havia tido uma integração explícita do RI da universidade com o marketing dos programas de pós-graduação: potenciais pós-graduandos podem avaliar o tipo de pesquisa e artigos/relatórios relacionados sendo produzidos por potenciais orientadores no processo da tomada de decisão. ${ }^{5}$

Relacionado ao papel de marketing do RI está seu uso para gerar indicadores de pesquisa (produtividade em pesquisa), utilizados em muitas universidades mundialmente para avaliar pesquisa e auxiliar na tomada de decisão em procedimentos profissionais de avaliação e progressão acadêmicas - Hélio Kuramoto cita o exemplo da Universidade de Liège, na Bélgica, onde o reitor, Prof. Bernard Rentier, "enfatizou que o depósito no RI daquela universidade era o único critério utilizado para a avaliação dos pesquisadores por aquela universidade" (KURAMOTO, 2011).

\section{A Realidade dos Repositórios Institucionais (RIs)}

Apesar dos esforços concentrados e integrados na implantação e na promoção de RIs no mundo todo, há um consenso inegável entre aqueles que pesquisam o AA de que os RIs são muito mais vazios do que o esperado e o desejável. Como Björk et al. (2008) e Hajjem et al. (2005) apontam, somente cerca de $15 \%$ dos 2,5 milhões de artigos publicados anualmente no mundo é autoarquivado nos RIs por seus autores. Harnard (2006) acrescenta que apenas

\footnotetext{
${ }^{4}$ Disponível em: <http://www.webometrics.info/>. Acesso em: 17 ago. 2011.

${ }^{5}$ No Liber Conference, Aarhus, Dinamarca, jun-jul, 2010.

InCID: R. Ci. Inf. e Doc., Ribeirão Preto, v. 2, n. 2, p. 160-177, jul./dez. 2011.
} 
15\% dos autores científicos autoarquivam seus artigos em RIs. Em 2007, Davis e Connolly (2007) observaram que, apesar do investimento institucional, o RI da Cornell University nos EUA estava sendo consideravelmente subutilizado pelo faculty staff de Cornell, afirmando que: "Embora exista um estrutura de RI instalada, a maioria se mantém em forma esqueletal, com muitas coleções vazias e esparsamente povoadas. [...] Há pouca evidência para sugerir que os pesquisadores individuais estão fazendo contribuições significativas de papers no repositório" (tradução nossa). De modo semelhante, relatando a experiência da implementação e da incorporação do RI na cultura institucional da Universidade de Minho, em Portugal, Ferreira et al. (2008) afirmam que mesmo que o RI tenha sido lançado em 2003 acompanhado por um programa integrado de advocacy, que até incluí um incentivo financeiro para o departamento do autor depositante,

Até o final de 2004, o número de documentos no RI alcançou em torno de 630. Sentiu-se que a pesar das várias conclamações para a submissão de trabalhos, não surtiram os efeitos esperados. O número de documentos autoarquivados ainda ficou baixo. Dos 630 documentos no repositório, somente 128 foram arquivados pelos próprios autores (tradução nossa).

Tudo isso está em nítido contraste com o crescimento contínuo do número de RIs sendo implantados mundialmente. O diretório de repositórios AA, OpenDOAR, mostra que o número de repositórios (que inclui bibliotecas digitais) quase dobrou entre 2005 e 2010 (OpenDOAR, 2010), alcançando mais de dois mil repositórios registrados no mês de julho de 2011.

Posto que a vantagem, muito divulgada, de RIs é que constituem uma potencial "vitrine" de marketing para uma instituição, o fato de os RIs ficarem relativamente vazios pode ser entendido como grave, as repercussões extrapolando meras especulações sobre o possível desinteresse dos pesquisadores em relação a AA e RIs. Como enfatizam Swan e Carr (2008, p. 32):

\footnotetext{
Exceto um número pequeno de instituições no mundo que têm repositórios grandes e em crescimento contendo artigos de pesquisas correntes (ao invés de apenas teses, literatura cinzenta ou literatura legada do passado), a maioria dos RIs são, de fato, vazios. Estão não apenas não elevando a visibilidade online da sua instituição, como também estão ativamente projetando uma imagem muito pobre da instituição no mundo. A vitrine está vazia (tradução nossa).
}

Um motivo apontado para explicar este cenário é a resistência em função de suas rotinas bastante atarefadas - Harnard (2006) menciona a "paralisia do Zeno" para explicar este fenômeno - ou da apatia dos pesquisadores em relação ao autoarquivamento em RIs e a 
sua falta de conhecimento sobre o AA em geral, possíveis motivos que serão abordados na seção seguinte.

\section{Possíveis Motivos para a Resistência de Autores}

Como mencionado anteriormente, o fato de que apenas $15 \%$ de todos os artigos publicados nas 24 mil revistas científicas está acessível em algum canal de AA parece ser paradoxal à luz da pesquisa que confirma que "a vasta maioria" de pesquisadores afirma que eles "voluntariamente"/"prontamente" fariam com que cópias dos seus artigos publicados fossem disponibilizados em RIs (SWAN; BROWN, 2004; SWAN, 2006). É interessante observar que os autores que já se consideram como "autores em AA" ranqueiam seu apoio para o princípio básico de abrir o acesso à pesquisa publicada mundialmente como seu principal motivo por serem a favor de AA - o conhecimento é visto como um "bem público"; também, eles acreditam que os periódicos em AA têm fluxos de trabalho de publicação mais ágeis e rápidos e, ainda, oferecem leitura mais ampla e maior impacto de citação (SWAN; BROWN, 2004).

Porém, outros pesquisadores também têm justificativas razoáveis por sua cautela em relação ao AA e aos RIs. Um motivo frequentemente citado por pesquisadores para explicar a sua resistência ao autoarquivamento num RI é a possibilidade de infringir a política de copyright da editora da revista na qual o artigo já foi publicado. No entanto, existem bases de dados autoritativas para ajudar autores a, em poucos passos, checarem os detalhes dos acordos de copyright, a RoMEO ${ }^{6}$, sendo a mais completa e atualizada destas bases. Cerca de $90 \%$ de todas as revistas científicas endossam algum tipo de submissão em AA, muitas das quais sem um período de embargo (RoMEO, 2010; HARNARD, 2006). Outras preocupações comuns expressas por pesquisadores que têm dúvidas em relação ao AA são que: i) o AA "passa por cima" do processo de revisão por pares e, assim, abriria a porta à publicação de baixa qualidade; ii) o AA acabará com a publicação de revistas científicas; iii) a submissão em um RI demandará tempo; iv) facilitará o plágio; e v) os autores serão obrigados a ceder os direitos de propriedade intelectual a sua universidade (KING et al., 2006; PINFIELD, 2004). Existe hoje uma superabundância de relatórios, websites e FAQs bem-fundamentados em pesquisas ${ }^{7}$

\footnotetext{
${ }^{6}$ RoMEO, disponível em: <http://www.sherpa.ac.uk/romeo/>. Existe a tradução em português da RoMEO. Também vide a base semelhante espanhola, Dulcinea, disponível em: <http://www.accesoabierto.net/dulcinea/> 
que respondem a este tipo de preocupação dada por pesquisadores como justificativa pelo não uso de um RI ou de uma revista em $\mathrm{AA}^{8}$.

\section{As Normas Científicas e o Sistema de Premiação (Reward System) na Cultura Científica}

Uma barreira substancial a aceitação e o uso maior de AA e de RIs que é difundida na comunidade científica é a percepção persistente do conteúdo em AA como sendo de qualidade inferior quando comparado a conteúdo das revistas científicas pagas via assinaturas - tollaccess (VAN WESTRIENEN; LYNCH, 2005). Isto aponta para a persistência da crença mais ardilosa e equivocada de que a literatura em AA não é literatura revisada por pares: como muitos pesquisadores no campo de AA têm enfatizado, a publicação neste âmbito não deveria ser equiparada com autopromoção por autores-pesquisadores ou "publicação de vaidade" (vanity publishing).

A importância do processo de revisão por pares para os pesquisadores deveria ser apreciada quando argumentando a favor de AA. Constitui uma das chaves do sistema de premiação (reward system) na academia, por sua vez baseado em normas que são crenças inculcadas quase que subliminarmente na comunidade científica global. O sociólogo Robert Merton definiu estas normas prescritivas como sendo: i) universalismo, ou seja, o desenvolvimento do estudo deveria focar nos critérios universais do objeto de estudo, e não nas particularidades do pesquisador, como reputação, nacionalidade e afiliação institucional; ii) "comunismo", que qualquer conhecimento surgido do empreendimento de pesquisa deveria se tornar público para o benefício da comunidade acadêmica inteira; iii) desinteresse, que a meta da pesquisa científica é a de buscar e contribuir para a verdade universal científica, sem ganhos ou interesses pessoais para os pesquisadores envolvidos; iv) ceticismo organizado, que significa que as afirmações de conhecimento feitas pelos pesquisadores serão examinadas e testadas antes de entrar no corpo de conhecimento científico compartilhado (MERTON, 1979). O processo de revisão por pares é baseado nas normas de comunismo e ceticismo organizado, no sentido de que o prêmio extrínseco para o pesquisador deriva do reconhecimento dos pares pela contribuição ao estoque comum de conhecimento. Porém, embora vise ser o mais objetivo possível, há muitos exemplos de subjetividade no processo de revisão de pares, sobretudo se não for completamente "cego". O próprio Merton (1988) observou que o

\footnotetext{
${ }^{8}$ Ver, por exemplo, <http://www.sherpa.ac.uk/documents/15concerns.html>. HARNARD, S. Self-arquiving FAQ. Disponível em: <http://www.eprints.org/openaccess/self-faq/>. Acesso em: 18 jul. 2011.

InCID: R. Ci. Inf. e Doc., Ribeirão Preto, v. 2, n. 2, p. 160-177, jul./dez. 2011.
} 
reconhecimento do trabalho científico por pares é frequentemente "enviesado a favor de cientistas estabelecidos" (MERTON, p. 607), um fenômeno que ele denominou "o efeito Mateus". ${ }^{9}$ Merton e seus pares evoluíram uma agenda de pesquisa na Sociologia da Ciência estudando esta "acumulação de vantagem" baseada na estratificação da ciência. Um resultado óbvio desta vantagem acumulada de reputação é que a quantidade de citações dos trabalhos do cientista renomado será maior: cifras de $0,3 \%$ de cientistas que publicam sendo citados mais que 100 vezes num dado período de tempo (de aproximadamente 20 anos) comparadas com $2,7 \%$ que são citados entre 25 e 100 vezes, e em torno de $58 \%$ citados apenas uma vez no mesmo período (GARFIELD apud MERTON, 1988, p. 611-612).

Ao longo dos anos, uma "vantagem acumulada" concomitante a certos títulos de revistas científicas se arraigou na comunidade científica pela aplicação do fator de impacto (Impact Factor - IF) para que os cientistas favoreçam certos periódicos em detrimento de outros, pois sabem que serão lidos e, portanto, citados mais ao publicar, por exemplo, em revista A que em B (GADD; OPPENHEIM, 2002). O IF da revista A é assegurado e preservado pela perpetuação desta prática bastante circular. Como um matemático entrevistado por Davis e Connolly (2007) observou: "Ser publicado [num certo periódico] transmite um carimbo de qualidade. Não tem nada a ver com a disseminação. Os periódicos também transmitem status, algo que o arXiv (repositório) não pode fazer, pelo menos, até hoje" (DAVIS; CONNOLLY, 2007).

Assim, a "moeda" do sistema de premiação na pesquisa científica é o reconhecimento "público" no sentido da propriedade intelectual ${ }^{10}$ da pesquisa pelos pares de uma dada área. Baseando-se na denominação notória de Beecher e Trowler (2001) das disciplinas científicas serem "tribos" operando dentro de "territórios", Paasi (2005, p. 773) observa que "o reconhecimento por pares e a liberdade tem sido reconhecidos como as forças primordiais na economia da ciência, não o dinheiro ou a segurança" (tradução nossa). E usualmente, serão os títulos de periódicos prestigiados daquele campo que os pesquisadores escolherão como o mais efetivo canal para obter o reconhecimento. Nesse sentido, como outro cientista entrevistado esclarece a Davis e Connolly (2007), o repositório será usado apenas se "for

\footnotetext{
${ }^{9}$ Do Novo Testamento, o Evangelho Segundo Mateus, 13:12 e 25:29: "Porque a todo que tiver será dado, e terá em abundância, mas daquele que não tem será tirado até o que ele tem".

${ }^{10}$ Pois como Merton (1988, p. 620) nota, “[...] é apenas um aparente paradoxo que, na ciência, a propriedade particular (do cientista) é estabelecida no ato de dar de graça a sua substância. Pois [...] somente quando os cientistas publicam seu trabalho e o fazem acessível, em geral, preferencialmente na publicação de artigos, monografias e livros que entrem nos acervos, é que é legitimamente estabelecido como mais ou menos seguramente da sua propriedade".
}

InCID: R. Ci. Inf. e Doc., Ribeirão Preto, v. 2, n. 2, p. 160-177, jul./dez. 2011. 
usado pelo resto da minha comunidade. Se um RI não aparece de modo regular numa busca, eu não colocaria meus papers nele”. Esta colocação confirma a visão de que os pesquisadores/cientistas são altamente apegados a sua disciplina ou ao seu campo e que "os avanços e a perícia baseados na disciplina constituem uma forma importante de credibilidade acadêmica" (DEEM, 2010, p. 39). De fato, nota-se com frequência que os acadêmicos e estudiosos usualmente têm uma aliança mais forte à sua disciplina/campo de estudo - sua "tribo" - do que a sua universidade/instituição.

É devido a este contexto de independência substancial em que a comunidade científica opera, e que permite total liberdade na determinação de onde publicar, que alguns concluem que a aceitação e o uso de canais de AA para a disseminação dos resultados de pesquisa apenas se darão por meio de medidas e políticas regulatórias e normativas.

\section{As Políticas para Encorajar o Uso de Canais de AA}

As políticas de Acesso Aberto podem ser localizadas em um ponto num espectro que se estende desde as afirmações gerais de apoio pelo, e promoção de, AA, até os mais prescritivos "mandatos" emitidos por órgãos de fomento à pesquisa, e, finalmente, a políticas específicas institucionais que declaram o apoio a AA como um princípio e encorajam os pesquisadores a publicarem nesses tipos de veículos ou a depositarem no RI, mas que pode também delinear os critérios para as metas gerais e operação do dia a dia de RIs.

$\mathrm{Na}$ primeira categoria, podemos citar as várias declarações e manifestos em apoio a AA que se disseminaram ao longo dos anos. A primeira delas foi a Budapest Open Access Initiative, de fevereiro de 2002, e que foi seguida pelos manifestos de Bethesda, de Berlin, pelas Nações Unidas e pela Association of College \& Research Libraries (ACRL), em 2003. ${ }^{11}$ Estas declarações constituem formas de conscientização dentro da comunidade acadêmica em torno da existência de caminhos de AA como a nova forma de comunicação científica, ao mesmo tempo em que dão uma credibilidade oficial, internacional e institucional ao movimento AA. Embora não sejam políticas a serem implementadas, seu valor reside na sua síntese dos argumentos principais a favor de AA, assim constituindo uma fundação inicial para as políticas de AA mais contextualmente específicas.

\footnotetext{
${ }^{11}$ Cf. http://www.soros.org/openaccess/initiatives.shtml>.

InCID: R. Ci. Inf. e Doc., Ribeirão Preto, v. 2, n. 2, p. 160-177, jul./dez. 2011.
} 
A segunda categoria, consistindo de mandatos de órgãos de fomento à pesquisa, constitui um instrumento importante de políticas: enquanto uma declaração exorta apoio e cumprimento, o mandato representa o braço executivo da política. Como tal, os mandatos são tidos como a política de AA do órgão de fomento. Eles estipulam que os pesquisadores, recebendo verba do órgão, devem subsequentemente disponibilizar seus artigos resultantes via canais em AA, ou publicar em periódicos AA ou via o autoarquivamento em RIs. A base de dados JULIET $^{12}$ registra detalhes sobre tais mandatos, que variam no que diz respeito sobre aquilo que estipulam: i) o autoarquivamento num repositório disciplinar ou num RI; ii) que o financiador da pesquisa cobriria os custos pela publicação numa revista AA; iii) que os termos do contrato com o órgão de fomento automaticamente precederia contratos futuros com editoras que restringem acesso, assim substituindo-os (SALE et al., 2010).

Em nível institucional, poderá haver uma política que apoia AA, ou um mandato, e também, para aquelas que tenham um RI, poderá haver uma política detalhada da operacionalidade do RI, que abordaremos na sequência. Os mandatos institucionais encorajam seus acadêmicos a depositarem manuscritos finais e revisados por pares no RI. Sale et al. (2010) argumentam que os mandatos institucionais são mais importantes que aqueles dos órgãos de fomento à pesquisa, principalmente porque nem toda a pesquisa é financiada, mas toda pesquisa é realizada no contexto de uma universidade ou de uma instituição de pesquisa. Mandatos institucionais precisam "caminhar de mãos dadas" com ações e posturas tangíveis, tais como respaldo e apoio institucional da alta administração, especialmente na forma de apoio à implantação de um RI. Uma política mais "micro" do próprio RI será necessária, que deve abranger tanto a missão global e os objetivos do RI (o "policy statement") quanto detalhar os critérios mais específicos para assegurar que os procedimentos de tomada de decisão relacionados aos aspectos rotineiros e operacionais do RI estejam sintonizados com a política mais global do RI. Por exemplo, se a missão do RI é a de "fazer livremente disponível as publicações científicas desta universidade em texto-completo", então isto teria que ser traduzido para o procedimento mais específico que controlaria o tipo de conteúdo depositado no RI, evitando o cenário de ter um RI povoado com apenas os metadados. ${ }^{13}$

\footnotetext{
12 Disponível em: <http://www.sherpa.ac.uk/juliet>. Ver também ROARMAP, em $<$ http://www.eprints.org/openaccess/policysignup/>.

${ }^{13}$ Ver <http://www.rsp.ac.uk/repos/checklist4> para um checklist de políticas para RIs. O OpenDOAR Policy Tool enumera um conjunto completo de opções para políticas de RIs, permitindo que um administrador do RI escolha os aspectos da política, e, com isso, gerar páginas web e documentos.
}

InCID: R. Ci. Inf. e Doc., Ribeirão Preto, v. 2, n. 2, p. 160-177, jul./dez. 2011. 


\section{O Conceito de Advocacy para o Acesso Aberto}

No uso anglo-saxônico do termo, advocacy significa recomendar, assegurar e defender uma certa posição, argumento ou grupo, usualmente representando os interesses daquele grupo. Os protagonistas de advocacy procuram o reenquadramento de questões, a reconfiguração do discurso predominante, a introdução de novas ideias e, ao fazer isso, “chamar a atenção e encorajar a ação" (KECK; SIKKINK, 1998, p. 217). Em um nível, as atividades de advocacy podem focar principalmente chamar a atenção, explicar, esclarecer dúvidas sobre novas práticas de disseminação a serem adotadas por pesquisadores que publicam. Tais iniciativas de advocacy são a jusantes (downstream), no sentido que focam os indivíduos em nível cognitivo, vendo-os acima de tudo como aqueles que tomam decisões racionais, operando num contexto no qual podem individualmente tomar decisões, supostamente livres de fatores influentes do contexto no qual trabalham. As campanhas informacionais usualmente operam nesse nível.

Se o ambiente institucional e suas demandas operam para induzir, facilitar e até "fossilizar" certos hábitos e práticas, então é possível, como afirmam Verplanken e Wood (2006), que as mudanças naquele ambiente - no "contexto do desempenho do hábito" ("habit performance context") - também possam facilitar mudanças no comportamento habitual e estabelecido. Nesse sentido, a advocacy a montante (“upstream”) será mais efetiva. Este tipo de intervenção focaliza:

[...] nas condições infra-estruturais mais amplas nas quais os comportamentos das pessoas são incorporados. Assim, as intervenções montantes podem consistir em incentivos econômicos, legislação, ou mudanças estruturais no ambiente de desempenho (performance environment). Estas intervenções visam fornecer contextos e estruturas societais que promovam e sustentem o comportamento desejado (ibid, p.95-96).

A importância atribuída às transformações no contexto para suscitar mudança de comportamento também foi destacada por Beer e Eisenstat (1990) quando analisando a transformação organizacional. Estes autores notaram que existe uma falácia subjacente a muitos programas de mudança que é a que o conhecimento e as atitudes nos indivíduos precisam ser mudados primeiramente, o que levaria a modificações no comportamento, que por sua vez, suscitará modificação mais ampla. Acrescentam que invertendo estas suposições mais provavelmente suscitaria mudança de comportamento: 


\begin{abstract}
A forma mais efetiva de mudar comportamento é a de colocar as pessoas num novo contexto organizacional que impõe nelas estes novos papéis, responsabilidades e relações. Este cria uma situação que, num dado sentido, "força" novas atitudes e comportamentos nas pessoas (BEER; EISENSTAT, 1990, p. 159).
\end{abstract}

De novo, a relevância deste cenário para a advocacy de AA e os RIs fica evidente: tem sido heuristicamente observado que "apenas os folders de informação não funcionam, não importa o quão chamativos sejam" ${ }^{\prime 4}$. O trabalho de advocacy do tipo político, networking e lobbying - com os protagonistas significativos e chaves como administradores, pró-reitores, representantes das agências de fomento à pesquisa, políticos - que visa atingir mudanças estruturais de mais longo prazo e mais aprofundadas, institucional e inter-institucionalmente, é cada vez mais entendido como a forma de avançar no domínio de publicação em AA e dos RIs.

\title{
De Advocacy a Jusante para Advocacy a Montante
}

Não estamos alegando aqui que as audiências-alvo são impermeáveis às iniciativas e ações de promoção a jusante, mas que dado o contexto no qual os pesquisadores-autores trabalham, e que o status quo institucional pode constituir uma barreira substancial à mudança no sentido que facilita e até incentiva a continuação de hábitos consagrados, tais ações a jusantes por elas só, apesar de serem informativas, exercerão um impacto limitado/restrito.

Para Verplanken e Wood (2006), os programas de advocacy montantes que têm como sua meta as ações institucionais que mudam o contexto serão mais efetivos em suscitar a desejada ruptura com os hábitos convencionais e profundamente arraigados (neste caso, de publicação), precisamente porque tais rupturas procuram alterar as "deixas" (incentivos) do contexto institucional que perpetuam os antigos modos e fomentam e apoiam novas formações de hábitos. As iniciativas de advocacy a jusante ainda têm um lugar para informar e motivar os indivíduos, que podem daí também potencialmente se tornarem "campeões" pela causa, mas o objetivo último é para tais iniciativas a serem expandidas, com o auxílio de tomadores de decisão-chave, em iniciativas a montantes mais amplas e de mais longo prazo. ${ }^{15}$

\footnotetext{
${ }^{14}$ Paráfrase de uma comunicação pessoal feita por um colega no Centre for Research Communications, University of Nottingham, Inglaterra.
}

InCID: R. Ci. Inf. e Doc., Ribeirão Preto, v. 2, n. 2, p. 160-177, jul./dez. 2011. 


\section{Considerações Finais: Mudança de Cultura na Academia Visando o Acesso Aberto}

A cultura organizacional é feita de normas, valores, filosofia, sentimentos e comportamento (SMIT; CRONJE apud MARTINS; TERBLANCHE, 2003). As intervenções do chamado "change management" para promover o AA terão que englobar estas facetas da cultura junto com a estrutura organizacional, os processos de trabalho e a infraestrutura física e das tecnologias de informação e comunicação (WORREN; RUDDLE; MOORE, 1999). De modo semelhante, como amplamente relatado na literatura, a participação dos stakeholders na escolha de intervenções é ideal para garantir que a mudança desejada enraize (SAUNDERS, 2005; VAN SCHOOR, 2003).

A "resistência" documentada dos pesquisadores ao autoarquivamento AA não toma a forma de oposição vociferadora; melhor, é feita da continuação silenciosa de hábitos anteriores e bem estabelecidos de publicação, e frequentemente do desconhecimento dos objetivos de AA. Os proponentes do AA na academia precisam ver tal "resistência" como uma oportunidade de focalizar e refinar seus argumentos a favor de AA. Isto é, de entender tal "resistência" como aspecto positivo, até como uma fonte de inovação para a implementação da mudança que está sendo proposta. Como Waddell e Sohal (1998, p. 545) afirmam:

\footnotetext{
Onde há resistência, há necessidade de examinar mais de perto os problemas que existem e considerar mais profundamente as mudanças propostas. [...] A resistência também encoraja a busca por métodos e resultados alternativos para sintetizar as opiniões conflitantes que possam existir. Assim, a resistência se torna uma fonte crítica de inovação num processo de mudança na medida em que mais possibilidades são consideradas e avaliadas (tradução nossa).
}

A implementação efetiva de uma política de AA - seja ela em escala institucional, regional, nacional ou internacional - dependerá da advocacy a montante, inovadora, para angariar o apoio "político" para o AA de cima para baixo, da alta administração da instituição, e com o apoio, de baixo para cima, de ambos: pesquisadores-autores e usuários finais da informação. 


\section{Referências}

ANTELMAN, K. Do Open-Access articles have a greater research impact? College \& Research Libraries News, v. 65, n. 5, p. 372-382, 2004. Disponível em: $<$ http://eprints.rclis.org/bitstream/10760/5463/1/do_open_access_CRL.pdf>. Acesso em: 25 ago. 2011.

BEECHER, T.; TROWLER, P. R. Academic tribes and territories. 2.ed. Buckingham, UK: Open University Press, 2001.

BEER, M.; EISENSTAT, R. A.; Spector, B. Why change programs don't produce change. In: Harvard Business Review. n. 68, p. 158-166, 1990.

BERLIN declaration on open access to knowledge in the Sciences and Humanities. 2003. Disponível em: <http://oa.mpg.de/openaccess-berlin/berlindeclaration.html>. Acesso em: 20 ago. 2011.

BETHESDA statement on open access publishing. 2003. Disponível em: <http://www.earlham.edu/ peters/fos/bethesda.htm>. Acesso em: 20 ago. 2011.

BJÖRK, B. et al. Open Access to the scientific journal literature: situation 2009. PLoS ONE v. 5, n. 6, e11273. Disponível em:

$<$ http://www.plosone.org/article/info:doi/10.1371/journal.pone.0011273>. Acesso em: 25 ago. 2011.

BUDAPEST OA Initiative. 2002. Disponível em:

<http://www.soros.org/openaccess/read.shtml> Acesso em: 20 ago.2011.

DAVIS, P.; CONNOLLY, M. Evaluating the reasons for non-use of Cornell University's installation of DSpace. D-Lib Magazine. v.13, n.3/4, 2007. Disponível em:

<http://www.dlib.org/dlib/march07/davis/03davis.html>. Acesso em: 25 ago. 2011.

DEEM, R. Herding the academic cats. Perspectives: Policy and Practice in Higher Education. v.14, n. 2, p. 37-43, 2010.

FERREIRA, M. et. al. Carrots and sticks: some ideas on how to create a successful institutional repository. D-Lib Magazine. v.14, n.1/2, 2008. Disponível em:

<http://www.dlib.org/dlib/january08/ferreira/01ferreira.html>. Acesso em: 22 ago. 2011.

GADD, E.; OPPENHEIM, C. Self-archiving - the 'right' thing? An introduction to the RoMEO Project. SCONUL Newsletter, n.27, p.34-37, Winter 2002. Disponível em: < http://www.sconul.ac.uk/publications/newsletter/27/ARTICLE9.PDF>. Acesso em: 20 ago. 2011.

GARFIELD, E. Citation indexes to science: a new dimension in documentation through association of ideas. Science. v.122, n. 3159, p.105-111, 1955. Republicado em: Essays of an Information Scientist, v.6, p. 468-471, 1983. Disponível em:

<http://garfield.library.upenn.edu/essays/v6p468y1983.pdf>, Acesso em: 18 ago. 2011. 
HAJJEM, C. et. al. Open Access to research increases citation impact. Technical Report. Institut des sciences cognitives Université du Québec à Montréal (submitted), 2005. Disponível em: <http://eprints.ecs.soton.ac.uk/11687>. Acesso em: 10 ago. 2011.

HARNARD, S. Opening access by overcoming Zeno's paralysis. In: JACOBS, N. (Ed.). Open Access: Key Strategic, Technical and Economic Aspects. Oxford:Chandos, 2006. Disponível em:

$<$ http://citeseerx.ist.psu.edu/viewdoc/download?doi=10.1.1.114.7787\&rep=rep1\&type=pdf $>$. Acesso em: 25 ago. 2011.

HARNARD, S. et.al. The green and the gold roads to Open Access. Nature Web Focus. Tues. 14 September, 2004. Disponível em: <http://eprints.ecs.soton.ac.uk/9940/>. Acesso em: 10 ago. 2011.

HARNARD, S. Publish or Perish - Self-Archive to Flourish: The Green Route to Open Access. ERCIM News, n. 64, 2006. Disponível em: <http://eprints.ecs.soton.ac.uk/11715/>. Acesso em: 08 ago. 2011.

HITCHCOCK, S. The effect of open access and downloads ('hits') on citation impact: a bibliography of studies. Disponível em: <http://opcit.eprints.org/oacitation-biblio.html>. Acesso em: 17 ago. 2011.

HOUGHTON, J. et al. Economic implications of alternative scholarly publishing models: Exploring the costs and benefits. Project Report. 2009. Disponível em: $<$ http://ierepository.jisc.ac.uk/278/>. Acesso em: 15 ago. 2011.

KECK, M; SIKKINK, K. Activists beyond borders. Advocacy networks in international politics. Ithaca: Cornell University Press, 1998.

KING, C. et. al. Scholarly Communication: academic values and sustainable models. Center for Studies in Higher Education, University of California, 2006. Disponível em: $<$ http://escholarship.org/uc/item/4j89c3f7>. Acesso em: 10 ago. 2011.

KURAMOTO, H. Blog do Kuramoto. Disponível em: <http://kuramoto.wordpress.com/>. Acesso em: jul. 102011.

KURAMOTO, H. Segredo para o sucesso na implantação de um RI. 28 jul. 2011. Disponível em: <http://kuramoto.wordpress.com/>. Acesso em: 20 ago. 2011

LAWRENCE, S. Free online availability substantially increases a paper's impact. Nature, 31 May 2001. Disponível em: <http://www.nature.com/nature/debates/eaccess/Articles/lawrence.html>. Acesso em: 10 jul. 2011.

MARTINS, E.; TERBLANCHE, F. Building organisational culture that stimulates creativity and innovation. European Journal of Innovation Management, v.6, n.1, p.64-74, 2003.

MERTON, R. K. The normative structure of Science. In: MERTON, R. K. The Sociology of Science: theoretical and empirical investigations. Chicago: University of Chicago Press, 1979, p. 267-278. 
MERTON, R. The Mathew effect in Science II. Cumulative advantage and the symbolism of intellectual property. ISIS, v. 79, p. 606-623, 1988.

NICHOLAS, D.; HUNTINGTON, P.; ROWLANDS, I. Open access journal publishing: the views of some of the world's senior authors. Journal of Documentation. v.61, n.4, p.497 $519,2005$.

NECOBELAC - A network of collaboration between Europe and Latin American Caribbean (LAC) countries. Deliverable D 3.3. Advocacy materials for local customisation and use within LAC countries. 2010. Disponível em:

$<$ http://www.necobelac.eu/documents/NECOBELAC_Deliverable_3_3_Final.pdf $>$. Acesso em: 31 ago. 2011.

OpenDOAR Directory of Open Access Repositories Available. Disponível em: $<$ http://www.opendoar.org/countrylist.php>. Acesso em: 05 jul. 2011.

PAASI, A. Globalisation, academic capitalism, and the uneven geographies of international journal publishing spaces. Environment and Planning A. v.37, p. 769-789, 2005.

PINFIELD, S. Self archiving publications. In: GORMAN, G. E.; ROWLAND, F. (eds.). International yearbook of Library and Information Management 2004-2005: scholarly publishing in an electronic era. London: Facet, 2004, p. 118-145. Disponível em: $<$ http://eprints.nottingham.ac.uk/142/>. Acesso em: 10 jul. 2011.

RoMEO. Disponível em: <http://www.sherpa.ac.uk/romeo/> Acesso em: 05 jul. 2011.

SALE, A; et. al. Open Access Mandates and the "Fair Dealing" Button. 2010. Disponível em: <http://arxiv.org/PS_cache/arxiv/pdf/1002/1002.3074v1.pdf>. Acesso em: 02 jun. 2010.

SAUNDERS, M. Opinion piece. Academy Exchange. n.2, p. 23, Autumn 2005.

SUBER, P. Open access overview. Focusing on open access to peer-reviewed research articles and their preprints. (Última revisão: novembro de 2010). Disponível em: $<$ http://www.earlham.edu/ peters/fos/overview.htm>. Acesso em: 04 jun. 2011.

SWAN, A. The culture of Open Access: researcher's views and responses. In: JACOBS, N., (Eds.) Open Access: key strategic, technical and economic aspects. Oxford: Chandos, 2006, p. 52-59. Disponível em: < http://eprints.ecs.soton.ac.uk/12428>. Acesso em: 20 ago. 2011.

SWAN, A. The Open Access citation advantage. Studies and results to date. Technical Report, School of Electronics \& Computer Science, University of Southampton. 2010. Disponível em: <http://eprints.ecs.soton.ac.uk/18516/> Acesso em: 20 ago. 2011.

SWAN, A.; BROWN, S. Authors and open access publishing. Learned Publishing, v. 17, p. 219-224, 2004. Disponível em:

$<$ http://eprints.ecs.soton.ac.uk/11003/1/Authors_and_open_access_publishing.pdf>. Acesso em: 20 ago. 2011.

SWAN, A.; BROWN, S. JISC/OSI journal authors survey report. 2005. Disponível em: <http://www.jisc.ac.uk/uploaded_documents/JISCOAreport1.pdf>. Acesso em: 20 ago. 2011.

InCID: R. Ci. Inf. e Doc., Ribeirão Preto, v. 2, n. 2, p. 160-177, jul./dez. 2011. 
SWAN, A.; CARR, L. Institutions, their repositories and the web. Serials Review, v.34, n.1, p.31-35, 2008.

VAN SCHOOR, A. Learning to overcome resistance to change in higher education: the role of Transformational Intelligence in the process. 2003. Disponível em:

<http://www.herdsa.org.au/wp-content/uploads/conference/2003/PDF/HERDSA16.pdf>. Acesso em: 20 ago. 2011.

VAN WESTRIENEN, G; LYNCH, C.. Academic institutional repositories. Deployment status in 13 nations as of mid. set. 2005. D-Lib Magazine, v.11, n.9, 2005. Disponível em: $<$ http://dlib.org/dlib/september05/westrienen/09westrienen.html>. Acesso em: 21 ago. 2011.

VERPLANKEN, B.; WOOD, W. Interventions to Break and Create Consumer Habits. Journal of Public Policy and Marketing. v.25, n.1, p. 90-103, 2006.

WADDELL, D; SOHAL, A. Resistance: a constructive tool for change management. Management Decision. v.36, n.8, p. 543-548, 1998. Disponível em:

$<$ http://www.icti.ie/articles/Resistance\%20a\%20constructive\%20tool\%20for\%20change\%20 management.pdf >. Acesso em: 20 ago. 2011.

WORREN, N; RUDDLE, K; MOORE, K. From organizational development to change management: the emergence of a new profession. Journal of Applied Behavioral Science. 35, n.3, p. 273-286, 1999. 